\title{
INJECTIVITY, PROJECTIVITY, AND THE AXIOM OF CHOICE \\ BY
}

\author{
ANDREAS BLASS
}

\begin{abstract}
We study the connection between the axiom of choice and the principles of existence of enough projective and injective abelian groups. We also introduce a weak choice principle that says, roughly, that the axiom of choice is violated in only a set of different ways. This principle holds in all ordinary Fraenkel-Mostowski-Specker and Cohen models where choice fails, and it implies, among other things, that there are enough injective abelian groups. However, we construct an inner model of an Easton extension with no nontrivial injective abelian groups. In the presence of our weak choice principle, the existence of enough projective sets is as strong as the full axiom of choice, and the existence of enough free projective abelian groups is nearly as strong. We also prove that the axiom of choice is equivalent to "all free abelian groups are projective" and to "all divisible abelian groups are injective."
\end{abstract}

The classical development of homological algebra [4] depends upon two theorems asserting that every module, over an arbitrary ring, is both a homomorphic image of a projective module and a submodule of an injective module. The usual proofs of these two theorems make use of the axiom of choice. Our purpose here is to investigate the connection between the axiom of choice and the principles of existence of enough projectives and enough injectives. In very rough terms, our results can be summarized by saying that the existence of enough injectives is a very weak form of choice, while the existence of enough projectives is rather strong.

In $\S 1$, we establish our notation and some preliminary results, for example that it suffices to consider abelian groups instead of modules over an arbitrary ring. $\$ 2$ is devoted to the proof that the axiom of choice is equivalent to key ingredients of the usual proofs of the theorems that there are enough projective and enough injective abelian groups. Thus, any proofs of these two theorems using less than the full strength of the axiom of choice would have to proceed along different lines. In $\$ 3$, we construct a model of set theory with no nontrivial injective abelian groups. It is a permutation model in which the atoms (= urelements) form a proper class; the same result can be obtained without atoms by means of forcing with a proper class of conditions. The need for proper classes in the construction of $\$ 3$ is shown in the next two sections. In $\S 4$, we isolate a property that distinguishes models

Received by the editors March 6, 1978.

AMS (MOS) subject classifications (1970). Primary 02K20 (= 04A25); Secondary 02K05, 02K 15, 13C10, $18 \mathrm{G} 05$. 
constructed with a set of atoms or forcing conditions from models that require a proper class of atoms or forcing conditions; this property is expressed by an axiom, which we call SVC, and which says roughly that the violations of the axiom of choice in the model are small, i.e., set-sized. In $\$ 5$ we prove from SVC that there are enough injective abelian groups. It follows that the existence of enough injective abelian groups is a very weak principle in that it does not imply any of the numerous weak forms of choice that can be violated in models built with a set of atoms or forcing conditions. The final two sections deal with projectivity; the results here are less satisfying than for injectivity. Unlike injectivity, projectivity is a nontrivial concept even in the category of sets, if the axiom of choice fails. $\$ 6$ is concerned with the principle that every set is the surjective image of a projective set. We show that this principle in conjunction with SVC implies the axiom of choice, so it is not deducible from any of the usual weak forms of choice since these can hold while choice fails in certain models built with a set of atoms or forcing conditions. Finally, in $\$ 7$, we derive several consequences of the existence of enough projective abelian groups or of enough free projective abelian groups. In particular, the latter together with SVC implies choice if there are no atoms.

We assume that the reader is familiar with the basic facts about the axiom of choice, including the construction of models of set theory where this axiom is false, as presented in [10]. For the concepts from category theory that we use without explanation in $\$ 1$, see [14].

1. Preliminaries. Our basic system of set theory is ZFA, the ZermeloFraenkel theory without the axiom of choice, modified to allow atoms, i.e., elementless things other than the empty set. Unlike Jech [10], we do not assume that the set of all atoms exists. ZF is the unmodified Zermelo-Fraenkel theory; ZFC and ZFCA are the theories obtained from ZF and ZFA by adding the axiom of choice, AC. We occasionally mention proper classes, but only as a shorthand to avoid a more cumbersome formulation in terms of sets and atoms. For the details of the elimination of proper classes, see [11, Chapter 1].

An object $P$ in a category $C$ is projective if the functor $\mathbf{C}(\boldsymbol{P},-)$ preserves epimorphisms. This implies that every epimorphism to $P$ splits, and the converse is true provided $\mathrm{C}$ has pullbacks that preserve epimorphisms. All the specific categories we shall consider satisfy this proviso. Note that a projective object in the category Set of sets is a set $\boldsymbol{P}$ such that, for any $P$-indexed family $\left\{X_{p} \mid p \in P\right\}$ of nonempty sets, there is a choice function, i.e., a function $f$ on $P$ such that $f(p) \in X_{p}$ for all $p$. Thus, the axiom of choice is equivalent to the assertion that all sets are projective. A category $\mathbf{C}$ is said to have enough projectives, and we write EPC, if every 
object of $\mathbf{C}$ admits an epimorphism from a projective object.

Dually, an object $I$ of $\mathbf{C}$ is injective if the functor $\mathbf{C}(\ldots, I)$ sends monomorphisms to epimorphisms. This implies that every monomorphism with domain $I$ splits, and the converse holds in the categories we shall consider because these categories have pushouts that preserve monomorphisms. $\mathbf{C}$ has enough injectives, written EIC, if every object admits a monomorphism to an injective object. For example, EISet is true because all nonempty sets are injective.

It is well known [16] that EIR-Mod, where $R$-Mod is the category of left modules over the ring $R$, follows easily from EIAb, where $\mathbf{A b}=\mathbf{Z}-\mathbf{M o d}$ is the category of abelian groups. The following lemma is the general principle behind this fact.

LEMMA 1.1. (a) Let $U: \mathbf{A} \rightarrow \mathbf{B}$ be a faithful, epimorphism-preserving functor with a left adjoint. If EPB then EPA.

(b) Let $U: \mathbf{A} \rightarrow \mathbf{B}$ be a faithful, monomorphism-preserving functor with a right adjoint. If EIB then EIA.

Proof. It suffices to prove (a), since (b) is its dual. Let $L$ be the left adjoint of $U$, and let $X$ be any object of $\mathbf{A}$. By EPB, there is a projective object $P$ of $\mathbf{B}$ with an epimorphism $\pi: P \rightarrow U X$. By adjointness, we get a map $\hat{\pi}: L P \rightarrow X$. This $\hat{\pi}$ is the composite of $L \pi$ and the co-unit of the adjunction $\varepsilon: L U X \rightarrow X$. $L \pi$ is epi since $\pi$ is and $L$, being a left adjoint, preserves epis. $\varepsilon$ is epi because $U$ is faithful. So $\hat{\pi}$ is epi, and it remains to prove that $L P$ is projective. If $\alpha$ : $Y \rightarrow Z$ is any epimorphism in $\mathbf{A}$, then the function $\mathbf{A}(L P, \alpha): \mathbf{A}(L P, Y) \rightarrow$ $\mathbf{A}(L P, Z)$ is the composite of adjunction isomorphisms and $\mathbf{B}(P, U \alpha)$ : $\mathbf{B}(P, U Y) \rightarrow \mathbf{B}(P, U Z)$. But $U$ and $B(P, \ldots)$ preserve epimorphisms, so this function is epi.

Corollary 1.2. (a) If EPAb then EPR-Mod for all rings $R$. (b) If EIAb then EI $R$-Mod for all rings $R$. (c) If EPSets then EPAb.

Proof. The forgetful functor $U: R$-Mod $\rightarrow \mathbf{A b}$ is faithful, preserves both monomorphisms and epimorphisms, and has both a left adjoint ( $R$ $\left.\otimes_{\mathbf{z}}\right)_{)}$and a right adjoint $\left(\operatorname{Hom}_{\mathbf{z}}(R, \ldots)\right)$. The forgetful functor from $\mathbf{A b}$ to Sets is faithful, preserves epimorphisms, and has a left adjoint (free abelian group). Thus, the corollary follows immediately from the lemma.

In view of this corollary, we shall not consider the general principles "EP $R$-Mod for all $R$ " and "EI $R$-Mod for all $R$ " but rather the equivalent special cases EPAb and EIAb.

2. Two algebraic equivalents of the axiom of choice. The usual proof of EPAb [4, §I.2] consists of two major steps. First, every abelian group is a homomorphic image of a free one. Second, all free abelian groups are 
projective. Similarly, the usual proof of EIAb [4, §I.3] consists of two major steps. First every abelian group is a subgroup of a divisible one. Second, all divisible abelian groups are injective.

In both cases, the first step can be carried out in ZFA. For the projective case, this is clear, since the proof in [4] does not use choice at all. (Any abelian group is the image of the free group on its underlying set, via the co-unit of the free-underlying adjunction, and this co-unit is epi because the underlying-set functor is faithful.) For the injective case, it is slightly less clear, since the proof in [4] uses a nonzero ordinal number $\Omega$ into which the ground ring ( $Z$ in our case) cannot be mapped cofinally; it is not known whether the existence of such an ordinal is provable in ZFA. However, a closer inspection of the proof in [4] shows that the following weaker condition on $\Omega$ is sufficient: Every ideal has a set of generators which cannot be mapped cofinally into $\Omega$. Since $\mathbf{Z}$ is a principal ideal domain, this condition is satisfied by the first infinite ordinal $\omega$.

On the other hand, the second step is, in both cases, equivalent to the axiom of choice. This result was announced in [3]. It includes an answer to a question of Armbrust [2].

THEOREM 2.1. The following are equivalent (in ZFA).

(a) Every free abelian group is projective.

(b) Every divisible abelian group is injective.

(c) The axiom of choice.

Proof. The usual proofs of (a) and (b) can clearly be formalized in ZFCA, so (c) implies (a) and (b). For the converse implications, it is convenient to introduce an auxiliary statement.

(d) For any family $\left\{A_{i} \mid i \in I\right\}$ of sets $A_{i}$ with at least two elements each, there is a family $\left\{B_{i} \mid i \in I\right\}$ such that each $B_{i}$ is finite and $\varnothing \neq B_{i} \subsetneq A_{i}$.

The plan of our proof is to show (a) $\rightarrow(d),(b) \rightarrow(d)$, and (d) $\rightarrow(c)$.

(d) $\rightarrow$ (c): Given a family $\left\{X_{j} \mid j \in J\right\}$ of nonempty sets, we seek a choice function. Let $X=\cup_{j \in J} X_{j}$, and apply (d) to the family of all subsets of $X$ that have at least two elements. We obtain a function $f$ that assigns to each such set $A$ a finite nonempty proper subset $f(A)$ of $A$. For each $j$, consider the sequence $X_{j}, f\left(X_{j}\right), f^{2}\left(X_{j}\right), \ldots$, where we continue to apply $f$ as long as we have a set with at least two elements. Since the sets in this sequence are strictly decreasing (because $f(A) \subsetneq A$ ) and all but (possibly) the first are finite, the sequence must terminate. The only way it can terminate is for some $f^{n}\left(X_{j}\right)$ to have fewer than two elements; since $f^{n}\left(X_{j}\right)$ is nonempty, it must be $\left\{x_{j}\right\}$ for some $x_{j} \in X_{j}$. Sending $j$ to $x_{j}$, we have the required choice function.

(For readers thoroughly familiar with [10], we mention a shorter but far less direct proof of $(d) \rightarrow(c)$. Clearly, (d) implies both the selection principle and 
multiple choice. The former implies that every set can be linearly ordered $[10$, p. 53], and the latter implies that every linearly ordered set can be well-ordered [10, p. 133].)

(a) $\rightarrow$ (d): Given $\left\{A_{i} \mid i \in I\right\}$ as in (d), let $F$ be the free abelian group on $I$, and let $G$ be the free abelian group on the disjoint union

$$
\coprod_{i \in I} A_{i}=\left\{(i, a) \mid i \in I, a \in A_{i}\right\} .
$$

The projection from $\amalg_{i \in I} A_{i}$ to $I$, sending $(i, a)$ to $i$, extends to a homomorphism $\pi: G \rightarrow F$, which is surjective because none of the $A_{i}$ are empty. By (a), $F$ is projective, so $\pi$ splits; let $\sigma: F \rightarrow G$ be a section of $\pi$. For each $i \in I, \sigma(i)$ contains terms $(i, a)$ (where $a \in A_{i}$ ) with integer coefficients adding up to 1 , because $\pi \sigma(i)=i$. (It may also contain terms $(j, a)$, where $a \in A_{j}$ and $j \neq i$, with integer coefficients adding up to 0 .) Define

$$
B_{i}=\left\{a \in A_{i} \mid(i, a) \text { has positive coefficient in } \sigma(i)\right\} \text {. }
$$

Since $\sigma(i)$ is a finite combination of generators of $G, B_{i}$ is finite. If $B_{i}$ were empty, the coefficients of the $(i, a)$ in $\sigma(i)$ would have a sum $\leqslant 0$. If $B_{i}$ were equal to $A_{i}$, this same sum would be at least the cardinal of $A_{i}$, which is $\geqslant 2$. Since in fact the sum is 1 , we have $\varnothing \neq B_{i} \subsetneq A_{i}$, as required.

(b) $\rightarrow$ (d): Let $\left\{A_{i} \mid i \in I\right\}$ be as in (d). Let $V$ be the rational vector space with basis $\mathrm{II}_{i \in I} A_{i}$, viewed as just an additive group. Let $D$ be the quotient of $V$ by the subgroup (not the rational subspace) generated by elements of the form $(i, a)-(i, b)$, where $i \in I$ and $a, b \in A_{i}$. Thus, for each $i$, all the elements $(i, a) \in V$, with $a \in A_{i}$, represent the same element $\hat{i}$ of $D$. More generally, an element $v \in V$ represents $\hat{i} \in D$ if and only if (a) all its coefficients (in the basis $\amalg_{i \in I} A_{i}$ ) are integers, and (b) the sum over $a \in A_{j}$ of the coefficients of $(j, a)$ is 1 if $j=i$ and 0 otherwise.

For each $i \in I$, let $m_{i}$ be the cardinality of $A_{i}$ if this is finite, and let $m_{i}=2$ if $A_{i}$ is infinite. Let $F$ be the free abelian group on $I$ and define homomorphisms

$$
\alpha: F \rightarrow D: i \mapsto \hat{i} \text { and } \mu: F \rightarrow F: i \mapsto m_{i} i .
$$

Since $V$ is divisible, so is $D$, and, by (b), $D$ is injective. Since $\mu$ is obviously monic, we have a homomorphism $\beta: F \rightarrow D$ such that $\alpha=\beta \circ \mu$, which means that $\hat{i}=m_{i} \beta(i)$.

Temporarily fix an arbitrary $i \in I$. For any $v \in V$ representing $\beta(i) \in D$ and for any $a \in A_{i}$, let $c_{v a}$ be the coefficient of $(i, a)$ in $v$. Since $m_{i} v$ represents $\hat{i}$, the $c_{v a}$ must be integers divided by $m_{i}$, and their sum is $1 / m_{i}$. Thus, not all the $c_{v a}$ are integers, though of course all but finitely many of them are zero. Let $n_{i}$ be the first positive integer $n$ such that, for at least one $a \in A_{i}, c_{v a}$ is congruent to $n / m_{i}$ modulo 1 . Thus $1 \leqslant n_{i} \leqslant m_{i}-1$. Let

$$
F_{i}=\left\{a \in A_{i} \mid c_{v a} \equiv n_{i} / m_{i} \bmod 1\right\}
$$


Note that $n_{i}$ and $F_{i}$ are independent of the choice of $v$, for the coefficients of any other $v^{\prime}$ representing $\beta(i)$ differ from the coefficients of $v$ by integers. Clearly, $F_{i}$ is a nonempty finite subset of $A_{i}$. It remains only to prove that $F_{i} \neq A_{i}$.

Suppose, for some $i, F_{i}=A_{i}$. So $A_{i}$ is finite, $m_{i}$ is the cardinality of $A_{i}$, and $c_{v a} \equiv n_{i} / m_{i} \bmod 1$ for all $a \in A_{i}$. But then $1 / m_{i}=\Sigma_{a \in A_{i}} c_{v a} \equiv\left(n_{i} / m_{i}\right) m_{i} \equiv$ $0 \bmod 1$, a contradiction.

Theorem 2.1 implies that any proof of EPAb or EIAb from principles weaker then $\mathrm{AC}$ must use a different construction of the required projective or injective groups than the usual proof. Such a proof of EIAb will be given in §; we do not know whether one exists for EPAb.

3. A model without injective abelian groups. We shall show that EIAb is independent of ZF by constructing a model of ZF in which there are no nontrivial injective abelian groups at all. For simplicity, we first construct a permutation model of ZFA having this property. Afterward, we give a forcing construction of a $\mathrm{ZF}$ model with the desired property. This independence result has also been obtained, independently, by W. Hodges [9].

THEOREM 3.1. The existence of a nontrivial injective abelian group is not provable in ZFA.

Proof. We begin with a universe $V$, satisfying ZFCA, in which the atoms form a set $A$ of cardinality $\aleph_{1}$. We fix a bijection between $A$ and $Z \times \aleph_{1}$ and use it to write

$$
A=\left\{a_{\xi}^{i} \mid i \in \mathbf{Z}, \xi \in \aleph_{1}\right\}
$$

For any $x \in V$, we define the base of $x$ to be $B(x)=A \cap T C(x)$, where $T C$ means transitive closure. We call $x$ small if its base is countable. (Although we do not need this fact, we point out that the small sets and atoms constitute an inner model of ZFCA in which there is no set of all atoms.)

For each $\xi \in \aleph_{1}$, let $g_{\xi}$ be the permutation of $A$ defined by

$$
g_{\xi}\left(a_{\eta}^{i}\right)= \begin{cases}a_{\eta}^{i+1} & \text { if } \eta=\xi, \\ a_{\eta}^{i} & \text { if } \eta \neq \xi,\end{cases}
$$

and let $G$ be the group of permutations generated by all the $g_{\xi}$ 's. For each finite set $E \subseteq \aleph_{1}$, let $H_{E}$ be the subgroup of $G$ generated by the $g_{\xi}$ 's with $\xi \notin E$. The groups $H_{E}$ generate a normal filter $\mathscr{F}$ of subgroups of $G$, and we define the notions of symmetric and hereditarily symmetric in the usual way [10, Chapter 4]. (Thus, the hereditarily symmetric sets constitute the Fraenkel-Mostowski model determined by $G$ with supports of the form $\left\{a_{\xi}^{i} \mid i \in \mathbf{Z}, \xi \in E\right\}$ with $E$ a finite subset of $\aleph_{1}$.) We deviate slightly from 
standard usage by calling $E$ a support of $x$ if $H_{E}$ fixes $x$; thus a support is a subset of $\aleph_{1}$, not of $A$.

Let $M$ be the class of small hereditarily symmetric sets and atoms. We shall show that $M$ is a model of ZFA in which the only injective abelian groups are the trivial groups.

Because of the restriction to small sets, $M$ is not a permutation model in the usual sense, so it is necessary to check that the axioms of ZFA are satisfied. Extensionality (in the form that permits atoms), regularity, and the axiom asserting that only sets have members are satisfied because $M$ is a transitive submodel of $V$. The axioms of null-set and infinity are satisfied because all ordinals have empty base and empty support. The axioms of union and power set are satisfied because $\cup x$ and $\mathcal{P}^{M}(x)$ have the same base and the same (or smaller) support as $x$. It remains to prove replacement in $M$. Suppose we have $x$ and $\mathbf{w}$ in $M$ and a formula $\phi$ such that

$$
(\forall u \in x)(\exists ! v \in M) \phi^{M}(u, v, \mathbf{w}) .
$$

We must show that the set

$$
y=\left\{v \in M \mid(\exists u \in x) \phi^{M}(u, v, \mathbf{w})\right\}
$$

is in $M$. (Here $w$ is a finite sequence of parameters, and $\phi^{M}$ is $\phi$ relativized to $M$.) As each member of $y$ is in $M$ by definition and as $y$ is fixed by all the $g_{\xi}$ 's that fix $\mathbf{w}$ and $x$, it is clear that $y$ is hereditarily symmetric, and it remains to prove that $y$ is small. Let $C$ be the set of $\xi \in \aleph_{1}$ such that some $a_{\xi}^{i}$ is in the base of $x$ or $\mathbf{w}$. Since $C$ is countable because $x, \mathbf{w}$ are small, the proof will be complete if we show that

$$
(\forall v \in y)\left(\forall a_{\xi}^{i} \in B(v)\right) \xi \in C,
$$

so suppose that the base of some $v_{0} \in y$ contains $a_{\xi}^{i}$ with $\xi \notin C$. Let $\eta$ be an arbitrary element of $\aleph_{1}-C-\{\xi\}$, and let $h$ be the permutation of $A$ that interchanges $a_{\xi}^{j}$ and $a_{\eta}^{j}$ for each $j \in \mathbf{Z}$ while fixing $a_{\zeta}^{j}$ for all $\zeta \neq \xi$, $\eta$. (It is true but irrelevant that $h \notin G$.) Using the equations $h g_{\xi} h^{-1}=g_{\eta}, h g_{\eta} h^{-1}=$ $g_{\xi}$, and $h g_{\zeta} h^{-1}=g_{\xi}$ for $\zeta \neq \xi, \eta$, one easily verifies that $h$ (extended to $V$ in the canonical way) sends symmetric sets to symmetric sets (sets supported by $E$ go to sets supported by $h(E))$. Since also $B(h(x))=h(B(x))$, it follows that $h$ maps $M$ onto $M$ and is in fact an automorphism of $M$. As $h$ fixes all the atoms in the bases of $x$ and $\mathbf{w}$ because $\xi, \eta \notin C$, it must fix $x$ and $\mathbf{w}$ themselves as well as all the elements $u$ of $x$ (for $B(u) \subseteq B(x)$ ). Since $v_{0} \in y$, there is $u_{0} \in x$ such that $v_{0}$ is the unique $v \in M$ satisfying $\phi^{M}\left(u_{0}, v, w\right)$. From $\phi^{M}\left(u_{0}, v_{0}, w\right)$ and the fact that $h$ is an automorphism of $M$ fixing $u_{0}$ and $w$, we infer that $\phi^{M}\left(u_{0}, h\left(v_{0}\right), w\right)$. By the uniqueness of $v_{0}, h\left(v_{0}\right)=v_{0}$. Leaving $v_{0}$ fixed, $h$ must also fix its base. Since this base contains $a_{\xi}^{i}$, it must also contain $h\left(a_{\xi}^{i}\right)=a_{\eta}^{i}$. There are uncountably many possible values of $\eta$, so 
we have shown that the base of $v_{0}$ is uncountable, contradicting the fact that $v_{0} \in M$. This contradiction completes the proof that $y \in M$, so $M$ satisfies replacement and thus is a model of ZFA.

To complete the proof, we consider an arbitrary injective (in the sense of $M)$ abelian group $I \in M$ and prove $I$ is trivial. Let $C$ be a countable subset of $\aleph_{1}$ containing all those $\xi$ such that some $a_{\xi}^{i} \in B(I)$ and containing infinitely many other $\xi$ s as well. Let $Q$ be the abelian group generated by the set $\left\{a_{\xi}^{i} \mid i \in \mathbf{Z}, \xi \in C\right\} \cup\{1\}$ subject to the relations $a_{\xi}^{i}+1=a_{\xi}^{i+1} . Q$ is small because its base is $\left\{a_{\xi}^{i} \mid i \in \mathbf{Z}, \xi \in C\right\}$ and $C$ is countable. $Q$ is symmetric because it is fixed by every $g_{\xi}$. Each element of $Q$, being an equivalence class of words in the generators, is hereditarily fixed by all $g_{\xi}$ except those finitely many whose indices $\xi$ occur as subscripts (in $a_{\xi}^{i}$ ) in every word of the equivalence class. (In $V, Q$ is freely generated by $\left\{a_{\xi}^{0} \mid \xi \in C\right\} \cup$ $\{1\}$, but this set of generators is not in $M$ because it is not symmetric.) Let $P$ be the infinite cyclic subgroup of $Q$ generated by 1 .

Consider an arbitrary $x \in I$. The injectivity of $I$ allows us to extend the homomorphism $P \rightarrow I$ sending 1 to $x$ to a homomorphism $\alpha: Q \rightarrow I$ in $M$. Choose $\xi \in C$ so that (a) no $a_{\xi}^{i}$ is in $B(I)$ and (b) $g_{\xi}$ fixes $\alpha$. This is possible because $C$ was chosen so that infinitely many $\xi \in C$ satisfy (a) and because, by symmetry of $\alpha$, all but finitely many $\xi$ satisfy (b). Let $q=\alpha\left(a_{\xi}^{0}\right)$. Then $q \in I$, so $B(q) \subseteq B(I)$, so no $a_{\xi}^{i}$ is in $B(q)$, so $g_{\xi}$ fixes $q$. Therefore,

$$
\begin{aligned}
q & =g_{\xi}(q) & & \\
& =g_{\xi}\left(\alpha\left(a_{\xi}^{0}\right)\right) & & \text { (definition of } q) \\
& =g_{\xi}(\alpha)\left(g_{\xi}\left(a_{\xi}^{0}\right)\right) & & \left(g_{\xi} \text { is an automorphism of } V(\text { or } M)\right) \\
& =\alpha\left(g_{\xi}\left(a_{\xi}^{0}\right)\right) & & (\text { condition }(\mathrm{b}) \text { on } \xi) \\
& =\alpha\left(a_{\xi}^{1}\right) & & \left(\text { definition of } g_{\xi}\right) \\
& =\alpha\left(a_{\xi}^{0}+1\right) & & (\text { defining relation of } Q) \\
& =\alpha\left(a_{\xi}^{0}\right)+\alpha(1) & & (\alpha \text { is a homomorphism) } \\
& =q+x & & \text { (definition of } q \text { and } \alpha) .
\end{aligned}
$$

Therefore, $x=0$. Since $x$ was an arbitrary element of $I, I$ is trivial.

As we shall see in $\$ \S 4$ and 5 , it was essential in the preceding proof that the set $A$, on which we can define and manipulate automorphisms be a proper class in the sense of $M$, although its size is only $\aleph_{1}$ in the "real" world $V$. The proof depended upon the availability of a $Q$ that is "bigger" than the injective $I$ in the sense that we have an automorphism $g_{\xi}$ of the universe that moves an element $a_{\xi}^{0}$ of $Q$ (in a controllable way) while fixing all elements of $I$. The device of restricting to small sets, thereby making the set $A$ a proper class, seems to have no analog for ZF models (without atoms). Thus, in order to 
transfer Theorem 3.1 to $\mathrm{ZF}$ by the usual method of replacing atoms with equivalence classes of generic sets [10, Chapter 6], we need to use, not $\boldsymbol{\aleph}_{1}$ generic sets, but a proper class of them. Apart from this change, the proof of the following theorem is the standard transfer of the proof of 3.1. Although inner models of Easton models have been used before to obtain independence results concerning the axiom of choice [15], there seems to be no published proof of such a theorem. Accordingly, we give the following proof in more detail than would otherwise be necessary for such a ZF transfer of a ZFA result.

THEOREM 3.2. The existence of a nontrivial injective abelian group is not provable in $\mathrm{ZF}$.

Proof. We begin with a ground model $L$, which, as the notation indicates, is assumed to satisfy the axiom of constructibility, in addition to ZFC. For each regular cardinal $\xi$ of $L$, we adjoin a $Z$-indexed family of generic sets, $b_{\xi}^{i} \subseteq \xi$ for $i \in \mathbf{Z}$, by Easton forcing (see [7] or [19, §12]). Thus, a forcing condition $p$ is a function into $\{0,1\}$ from a set $\operatorname{Dom}(p)$ of triples $(\xi, i, \gamma)$, where $\xi$ is a regular cardinal of $L, i \in \mathbf{Z}, \gamma<\xi$, and $\{(\xi, i, \gamma) \in \operatorname{Dom}(p) \mid \xi \leqslant$ $\eta\}$ has cardinality $<\eta$ for each regular cardinal $\eta$ of $L$. We call the resulting forcing extension $V$, and we think of it as the universe in what follows. Note that cardinals and cofinalities are preserved by the extension.

We define $a_{\xi}^{i}$ to be the set of all subsets of $\xi$ whose symmetric difference with $b_{\xi}^{i}$ is a constructible set of cardinality $<\xi$. We extend the language of ZF by adding a new unary (partial) function symbol $S$, which we interpret in $V$ by defining $S\left(a_{\xi}^{i}\right)=a_{\xi}^{i+1}$, with $S(x)$ undefined if $x$ is not $a_{\xi}^{i}$ for any regular $\xi$ and any $i \in \mathbf{Z}$. The new instances of the replacement scheme involving $S$ are satisfied in $V$, since it is a routine matter to define forcing and to prove all the usual facts about it for the extended language. (In fact, $V$ would satisfy replacement even if we added a symbol for the function $(\xi, i) \mapsto b_{\xi}^{i}$, from which $S$ is definable.)

Let $M$ be the submodel of $V$ consisting of sets that are hereditarily ordinal definable (HOD), in the extended language, from finitely many $b_{\xi}^{i}$,s. Then $M$ is a model of $\mathrm{ZF}$, and we shall show that all its injective abelian groups are trivial.

Suppose $I$ were an injective abelian group with a nonzero element $x$. Let $\kappa$ be a cardinal so large that every element of $I$ is HOD from $b_{\xi}^{i}$,s with $\xi<\kappa$ and so large that infinitely many regular cardinals $<\kappa$ do not occur as subscripts of $b$ 's used in these definitions. Let $Q$ be the abelian group generated by $\left\{a_{\xi}^{i} \mid i \in \mathbf{Z}, \xi<\kappa\right\} \cup\{1\}$ subject to the relations $a_{\xi}^{i}+1=a_{\xi}^{i+1}$. Let $P$ be the infinite cyclic subgroup generated by 1 . Note that $M$ contains $Q$, $P$, and the homomorphism $P \rightarrow I$ that sends 1 to $x$. As $I$ is injective in $M$, there is a homomorphism $\alpha: Q \rightarrow I$ in $M$ with $\alpha(1)=x$. By the choice of $\kappa$, 
we can find a regular cardinal $\xi<\kappa$ such that $\alpha$ and all elements of $I$ are ordinal definable in $V$ using $b$ 's with subscripts different from $\xi$. Fix such a $\xi$, and set $q=\alpha\left(a_{\xi}^{0}\right) \in I$.

Take a formula defining $q$ in $V$, with ordinals and $b_{\eta}^{i}$ 's $(\eta \neq \xi)$ as parameters. Replace the parameters with their canonical names in the forcing language to obtain a formula $\tilde{q}$, with one free variable, in the forcing language. Similarly, let $\tilde{x}, \tilde{\alpha}$, and $\tilde{a}_{\xi}^{i}$ be forcing language definitions of $x, \alpha$, and $a_{\xi}^{i}$ (where the last involves the name for $b_{\xi}^{i}$ but the others do not). The sentence

$$
\begin{aligned}
& (\exists u v w y z)\left[\tilde{q}(u) \wedge \tilde{x}(v) \wedge \tilde{\alpha}(w) \wedge \tilde{a}_{\xi}^{0}(y) \wedge \tilde{a}_{\xi}^{1}(z)\right. \\
& \qquad w \text { is a homomorphism } \wedge w(1)=v \wedge w(y)=u \\
& \qquad \wedge z=y+1 \wedge v \neq 0],
\end{aligned}
$$

whose meaning is clearer in the abbreviated form

$\dot{\alpha}$ is a homomorphism $\wedge \dot{\alpha}(1)=\dot{x} \wedge \dot{\alpha}\left(\dot{a}_{\xi}^{0}\right)=\dot{q}$

$$
\wedge \dot{a}_{\xi}^{1}=\dot{a}_{\xi}^{0}+1 \wedge \dot{x} \neq 0,
$$

is true in $V$, hence forced (over $L$ ) by some condition $p$ in the generic filter. Fix such a $p$.

We introduce two automorphisms, $g$ and $h$, of the class of forcing conditions as follows. For any condition $r$,

$$
\begin{aligned}
& g(r)(\xi, i, \gamma)=r(\xi, i-1, \gamma), \\
& g(r)(\eta, i, \gamma)=r(\eta, i, \gamma) \text { if } \eta \neq \xi
\end{aligned}
$$

where the equations are interpreted to mean that if either side is defined then so is the other and they are equal. Let $D$ be the set of triples at which $p$ and $g(p)$ are both defined and have different values. $D$ consists of fewer than $\xi$ triples, all having $\xi$ as the first component, and $D$ is constructible. Let

$$
h(r)(t)= \begin{cases}1-r(t) & \text { if } t \in D, \\ r(t) & \text { if } t \notin D .\end{cases}
$$

Thus, $h g(p)$ is compatible with $p$. Note that $g$, extended in the usual way to the forcing language, fixes $S$ and the name of $b_{\eta}^{i}$ for $\eta \neq \xi$ but sends the name of $b_{\xi}^{i}$ to that of $b_{\xi}^{i+1}$. Thus, it fixes $\tilde{q}, \tilde{x}$, and $\tilde{\alpha}$ but sends $\tilde{a}_{\xi}^{0}$ to $\tilde{a}_{\xi}^{1}$. Similarly, $h$ fixes the name of $b_{\eta}^{i}$ for $\eta \neq \xi$, sends the name of $b_{\xi}^{i}$ to the name of the symmetric difference $b_{\xi}^{i} \triangle D_{\xi}^{i}$ where $D_{\xi}^{i}=\{\gamma \mid(\xi, i, \gamma) \in D\}$, and sends $S$ to another name for $S$. (More precisely, the empty condition forces $h\left(\dot{b}_{\xi}^{i}\right)=$ $\dot{b}_{\xi}^{i} \triangle\left(D_{\xi}^{i}\right)^{\vee}$ and $\forall v(h(S)(v)=S(v))$.) It therefore fixes $\tilde{q}, \tilde{x}, \tilde{\alpha}$, and all $\tilde{a}_{\xi}^{i}$ (since $D_{\xi}^{i}$ is constructible and smaller than $\xi$ ). Since $p$ forces $\dot{\alpha}\left(\dot{a}_{\xi}^{0}\right)=\dot{q}$, we infer that $h g(p)$ forces

$$
\dot{\alpha}\left(\dot{a}_{\xi}^{1}\right)=\dot{q}
$$


But $p$ and $h g(p)$ are compatible, so there is a condition forcing both (1) and (2), which is absurd since (1) implies

$$
\dot{\alpha}\left(\dot{a}_{\xi}^{1}\right)=\dot{\alpha}\left(\dot{a}_{\xi}^{0}+1\right)=\dot{\alpha}\left(\dot{a}_{\xi}^{0}\right)+\dot{\alpha}(1)=\dot{q}+\dot{x} \neq \dot{q} .
$$

This contradiction completes the proof that all injective abelian groups in $M$ are trivial.

4. Small violations of choice. In this section, we begin an analysis of the need for proper classes of atoms or of forcing conditions in the constructions of the preceding section. We introduce the following weak form of the axiom of choice, which we call the axiom of small violations of choice (SVC) since it says that, in some sense, all the failure of choice occurs within a single set $S$.

SVC. There is a set $S$ such that, for every set $a$, there exist an ordinal $\alpha$ and a function from $S \times \alpha$ onto $a$.

Notice that the axiom would be unchanged if we replaced "from $S \times \alpha$ " with "from a subset of $S \times \alpha$ ", since the function could be extended to all of $S \times \alpha$ by making it constant outside its original domain, unless $a$ is empty in which case we just take $\alpha=0$. Likewise, we could change "onto $a$ " to "onto a superset of $a$ ". If $S$ is as required in SVC, we say that SVC holds with $S$. The manner in which SVC localizes the failure of choice within a set $S$ is indicated by the following theorem; the idea behind the proof of part (b) was shown to me, in a slightly different context, by David Pincus.

THEOREM 4.1. Assume SVC with $S$.

(a) AC holds if and only if there is a choice function for the nonempty subsets of $S$.

(b) Let $S^{<\omega}$ be the set of finite sequences of members of $S$. The Boolean prime ideal theorem holds if and only if there is an ultrafilter $U$ on $S<\omega$ that is regular in the sense that $\left\{p \in S^{<\omega} \mid s \in\right.$ range $\left.(p)\right\} \in U$ for every $s \in S$.

Proof. The "only if" assertions are both obvious. For the "if" part of (a), we use the choice function to well-order $S$, as in the standard proof that choice implies well-ordering. We obtain lexicographic well-orderings of $S \times$ $\alpha$ for all ordinals $\alpha$ and thus, by SVC, well-orderings of all sets. For the "if" part of (b), let $U$ be a regular ultrafilter on $S^{<\omega}$, and let $B$ be any Boolean algebra. By SVC, let $f$ map $S \times \alpha$ onto $B$. For each $p \in S^{<\omega}$, the subalgebra $B_{p}$ of $B$ that is generated by $f(\operatorname{range}(p) \times \alpha)$ admits a well-ordering (induced via $f$ by the standard ordering of $\alpha$ and the ordering of $\operatorname{range}(p)$ by $p$ ) and hence a prime ideal $I_{p}$, which is obtained uniformly from $p$. Then

$$
I=\left\{b \in B \mid\left\{p \in S^{<\omega} \mid b \in I_{p}\right\} \in U\right\}
$$

is easily seen to be a prime ideal in $B$.

The motivation for introducing SVC is that it seems to express the key 
property of "ordinary" permutation and symmetric models, built from a set of atoms or of forcing conditions. The next four theorems say that SVC holds in these models.

By a permutation model, we mean one obtained by the general method presented in Chapter 4 of [10]. Such a model is specified by giving a model $V$ of ZFCA in which the atoms form a set $A$, a group $\mathcal{G}$ of permutations of $A$, and a normal filter $\mathscr{F}$ of subgroups of $\mathcal{G}$. The permutation model then consists of the hereditarily symmetric elements of $V$.

THEOREM 4.2. Every permutation model satisfies SVC.

Proof. Let $M$ be the hereditarily symmetric submodel of $V$ determined by the group $\mathcal{G}$ and the filter $\mathscr{F}$. Let $\mathscr{F}^{\prime}$ be the set of groups $H \in \mathscr{F}$ that occur as the symmetry group $\operatorname{sym}(x)$ of some $x \in M$. For each $H \in \mathscr{F}^{\prime}$, choose (using $\mathrm{AC}$ in $V)$ an $x(H) \in M$ with $\operatorname{sym}(x(H))=H$. We assume that no permutation $g \in \mathcal{G}$ sends any $x(H)$ to any other $x\left(H^{\prime}\right)$; this can always be arranged by replacing $x(H)$ with $(x(H), \gamma)$, using different ordinals $\gamma$ for different $H$ 's. Let

$$
S=\left\{g(x(H)) \mid g \in \mathcal{G}, H \in \mathscr{F}^{\prime}\right\} .
$$

Each element of $S$ is in $M$, because $\mathcal{F}$ is normal, and $\operatorname{sym}(S)=\mathcal{G} \in \mathcal{F}$, so $S \in M$. We shall prove SVC with $S$. Let $a$ be any set in $M$; we must map a subset of $S \times \alpha$ onto $a$, for some ordinal $\alpha$.

Using AC in $V$, well-order $a$, and let $\alpha$ be its order-type. For any $H \in \mathscr{F}^{\prime}$ and any $\xi<\alpha$, let $y(\xi, H)$ be the $\xi$ th element $y \in a$ for which $\operatorname{sym}(y)=H$, if this $y$ exists; $y(\xi, H)$ is undefined otherwise. Let

$$
f=\left\{(g(x(H)), \xi, g(y(\xi, H))) \mid g \in \mathcal{G}, H \in \mathscr{F}^{\prime}, \xi<\alpha\right\} .
$$

All the elements of $f$ are obviously in $M$, and it is also obvious that $\operatorname{sym}(f)=\mathcal{G} \in \mathcal{F}$, so $f \in M$ (despite our use of a well-ordering of $a$ which may not be in $M)$. We shall show that $f$ is a function from a subset of $S \times \alpha$ onto $a$. If $(g(x(H)), \xi)=\left(g^{\prime}\left(x\left(H^{\prime}\right)\right), \xi^{\prime}\right)$, then $\xi=\xi^{\prime}$ and $x(H)=$ $g^{-1} g^{\prime}\left(x\left(H^{\prime}\right)\right)$. But we arranged, before defining $S$, that no element of $\mathcal{G}$ sends any $x(H)$ to another $x\left(H^{\prime}\right)$. Therefore $H=H^{\prime}$ and $g^{-1} g^{\prime} \in \operatorname{sym}(x(H))=H$ $=\operatorname{sym}(y(\xi, H))$. So $g^{\prime}\left(y\left(\xi^{\prime}, H^{\prime}\right)\right)=g^{\prime}(y(\xi, H))=g(y(\xi, H))$. This proves that $f$ is a function. It is onto $a$, because every element $y \in a$ is $y(\xi, H)=$ $f(x(H), \xi)$ for $H=\operatorname{sym}(y)$ and for some $\xi<\alpha$.

The set $\mathcal{F}^{\prime}$ used in this proof is a normal filter of subgroups of $\mathcal{G}$, and it determines the same permutation model as $\mathscr{F}$. It seems to be an open problem to give an intrinsic characterization of the filters that can occur as $\mathscr{F}^{\prime}$. The concept analogous to $\mathcal{F}^{\prime}$ in the context of Boolean-valued models was used by Grigorieff in [8, §8].

By a symmetric model, we mean one obtained by the general method 
presented in Chapter 5 of [10]. Such a model is specified by giving a ground model $M$ of ZFC, a complete Boolean algebra $B$ in $M$, an $M$-generic filter $G$ in $B$, a group $\mathcal{G}$ of automorphisms of $B$, and a normal filter of subgroups of $\mathcal{G}$. The symmetric model consists of the elements of $M[G]$ that hereditarily have symmetric names. (Note that, by Proposition 8.1.6 of [8], the elements that hereditarily have symmetric names are the same as those that have hereditarily symmetric names.)

THEOREM 4.3. Every symmetric model satisfies SVC.

Proof. We use the notation of the preceding paragraph. The class of elements of $M[G]$ having symmetric names is closed under ordinal definability in $M[G]$ (see [8, p. 480]) and includes $M$, so, by Theorem 7.4 .3 of [8], the model of sets hereditarily having symmetric names is $M[x]$ for some set $x \in M[G]$. It follows immediately that SVC holds with the set of finite sequences of elements of the transitive closure of $x$; see the proof of Theorem 4.5 below or [8, p. 451].

Instead of using symmetric submodels, some authors prefer to define submodels of generic extensions by taking the sets hereditarily ordinal definable over some particular set $A$. See, for example, $[19, \S 9]$. If the submodel so obtained includes $M$, it can be shown to satisfy SVC exactly as in the proof of Theorem 4.3. But even if it does not include $M$, it still satisfies SVC, as is shown by the following theorem, which makes no reference to the ground model $M$ or to forcing.

THEOREM 4.4. If $V$ is any model of ZF and $A \in V$, then the submodel $\mathrm{HOD}(A)$ of sets hereditarily ordinal definable over $A$ satisfies SVC.

In other "words", $\mathrm{ZF} \vdash \mathrm{SVC}$ HOD(A).

Proof. We work within a universe $V$ that satisfies ZF. We shall define a class $F$ of $V$ such that $F$ is a partial function from $S \times \operatorname{Ord}$ onto $\operatorname{HOD}(A)$ for a certain $S \in \operatorname{HOD}(A)$, and such that the only parameter in the definition of $F$ is $A$. Then the restrictions of $F$ to $S \times \alpha$, for arbitrary ordinals $\alpha$, will be in $\operatorname{HOD}(A)$ and will suffice to show $\operatorname{HOD}(A)$ satisfies $\mathrm{SVC}$ with $S$.

Let $B=(A \cup\{A\})^{<\omega}$, the set of finite sequences whose terms are $A$ or elements of $A$. We define a class $F_{1}$ that is a partial function from $B \times$ Ord onto $\operatorname{HOD}(A)$ by setting $F_{1}(b, \omega \cdot \alpha+n)$ equal to the unique element $y \in R_{\alpha}$ (the $\alpha$ th rank) which, together with the sequence $b$, satisfies, in $\left(R_{\alpha}, \in\right)$, the formula with Gödel number $n$. If there is not a unique such $y, F_{1}(b, \omega \cdot \alpha+$ $n$ ) is undefined. It is well known [19] that $F_{1}$ maps onto $\operatorname{HOD}(A)$, but readers who prefer to keep ordinal parameters in the "ordinal definitions" can redefine $F_{1}$ so that these parameters are coded along with $\alpha$ and $n$ in the second argument of $F_{1}$. If $B$ were in $\operatorname{HOD}(A)$, we would be finished. In most applications, this will be the case, but in general the members of members of 
$A$ need not be OD over $A$. Hence the following contortion.

For each ordinal $\alpha$, define an equivalence relation $\sim_{\alpha}$ on a subset of $B$, as follows. The field of $\sim_{\alpha}$ is the set of $b \in B$ for which $F_{1}(b, \alpha)$ is defined, and $b \sim_{\alpha} b^{\prime}$ if and only if $F_{1}(b, \alpha)=F_{1}\left(b^{\prime}, \alpha\right)$. Call two ordinals equivalent, $\alpha \equiv \beta$, if $\sim_{\alpha}$ and $\sim_{\beta}$ are the same, and call an ordinal minimal if it is the first ordinal in its equivalence class. Since $B$ is a set, there is only a set of possible relations $\sim_{\alpha}$, so there is an ordinal $\xi$ greater than all minimal ordinals. Let

$$
F=\left\{\left(F_{1}(b, \alpha), \beta, F_{1}(b, \beta)\right) \mid b \in B, \alpha \equiv \beta, \alpha \text { minimal }\right\} .
$$

We first observe that the class $F$ is a function, for if $F_{1}(b, \alpha)=F_{1}\left(b^{\prime}, \alpha^{\prime}\right)$ and $\beta=\beta^{\prime}$, with $\alpha \equiv \beta, \alpha^{\prime} \equiv \beta^{\prime}$, and both $\alpha$ and $\alpha^{\prime}$ minimal, then $\alpha \equiv \beta=\beta^{\prime} \equiv$ $\alpha^{\prime}$ and minimality require $\alpha=\alpha^{\prime}$, the equation $F_{1}(b, \alpha)=F_{1}\left(b^{\prime}, \alpha\right)$ requires $b \sim_{\alpha} b^{\prime}$, hence $b \sim_{\beta} b^{\prime}$ because $\alpha \equiv \beta$, and finally $F_{1}(b, \beta)=F_{1}\left(b^{\prime}, \beta\right)=$ $F_{1}\left(b^{\prime}, \beta^{\prime}\right)$. The range of $F$ is clearly the same as the range of $F_{1}$, namely $\operatorname{HOD}(A)$. The domain of $F$ is $S \times$ Ord, where

$$
S=\left\{F_{1}(b, \alpha) \mid b \in B, \alpha \text { minimal }\right\},
$$

which is clearly in $\operatorname{HOD}(A)$.

There is yet another definition of inner models violating $\mathrm{AC}$, relative constructibility. If $A$ is a transitive set, one puts $L_{0}(A)=A, L_{\alpha+1}(A)=$ set of parametrically definable subsets of $L_{\alpha}(A)$, and $L_{\lambda}(A)=\cup_{\alpha<\lambda} L_{\alpha}(A)$ for limit ordinals $\lambda$. Then $L(A)=\cup_{\alpha} L_{\alpha}(A)$ is an inner model of ZF. This construction is essentially the one used in Cohen's original proof [5] of the independence of $\mathrm{AC}$.

THEOREM 4.5. If $A$ is a transitive set in a model of ZF, then the submodel $L(A)$ satisfies SVC.

Proof. We work within a universe satisfying ZF. Introduce a ramified language that is like the language of $\mathrm{ZF}$ except that, first, instead of the usual quantifiers $\exists, \forall$, it has limited quantifiers $\exists^{\alpha}, \forall^{\alpha}$ whose intended interpretation is $\exists x \in L_{\alpha}(A), \forall x \in L_{\alpha}(A)$, second, it has limited comprehension operators which can be applied to a formula $\phi(x)$ to produce a term whose intended interpretation is $\left\{x \in L_{\alpha}(A) \mid L_{\alpha}(A) \vDash \phi(x)\right\}$, and, finally, it has constants for all the elements of $A$. (See [13] for details.) Then the elements of $L(A)$ are precisely the denotations of the closed terms of this language. The terms and formulas of the language can clearly be coded by pairs consisting of an ordinal and a finite sequence from $A$. Since the ramified language, its coding, and its "denotation" function (assigning to each term its denotation) are classes defined by formulas that are absolute for $L(A)$, it follows immediately that $L(A)$ satisfies SVC with $A^{<\omega}$.

To avoid possible confusion, we emphasize that, in the ramified language 
used in the preceding proof, the constants denoting elements of $A$ are to be obtainable from those elements (and vice versa) in an absolute manner; for example, the constant for $a$ could be $(0, a)$. These constants are thus quite different from the generic constants used by Cohen [5], since the latter are usually well-ordered even when their denotations are not. We also mention that, to fit Cohen's models into the form $L(A)$, one takes $A$ to be the set of denotations of the labels of level $<\alpha_{1}$, where $\alpha_{1}$ is as on p. 128 of [5].

This concludes our verification that the usual models of set theory without choice satisfy SVC. Notice that the models used in the proofs of Theorems 3.1 and 3.2 are not covered by this verification. The model of 3.1 is not covered by Theorem 4.2 because of the restriction to small sets in 3.1. The model of 3.2 is not covered by Theorem 4.4, because it consists of the sets HOD over a proper class. It will follow from Theorem 5.1 that these models, and indeed any models that serve the purposes of $\S 3$, do not satisfy SVC.

The following theorem will be used here only as a lemma in the proof of Theorem 4.7, but it seems to be of interest in its own right. By a generic extension of a model $M$, we mean a model $M[G]$ obtained by adjoining an $M$-generic filter $G$ in some notion of forcing $P \in M$. To avoid restricting ourselves to countable models, we allow $M[G]$ to be Boolean-valued.

THEOREM 4.6. $A$ model of $\mathrm{ZF}$ satisfies SVC if and only if some generic extension of it is a model of ZFC.

Proof. Suppose $M$ satisfies SVC with $S$. Let $P$ be the set of finite partial functions from $\omega$ to $S$, and let $G$ be an $M$-generic filter in $P$. The union of $G$ is, in $M[G]$, a function from $\omega$ onto $S$. Every set in $M$, being an image of $S \times \alpha$ for some ordinal $\alpha$, is thus well-orderable in $M[G]$. Just as in the usual proof of AC in generic extensions of ZFC models, we represent each set in $M[G]$ as the image of a set in $M$ and conclude that every set in $M[G]$ admits a well-ordering in $M[G]$.

Conversely, suppose $G$ is an $M$-generic filter in some notion of forcing $P \in M$ and $M[G]$ satisfies AC. For any set $a \in M$, there exist an ordinal $\alpha$ and a map $f \in M[G]$ of $\alpha$ onto $a$. Let $\dot{f}$ be a name for $f$ in the forcing language, and define, in $M$, a function $g$ on a subset of $P \times \alpha$ by

$$
g(p, \xi)=x \text { if } p \Vdash \dot{f} \text { is a function and } \dot{f}(\check{\xi})=\check{x} \text {. }
$$

For each $x \in a$, there is a $\xi<\alpha$ such that $f(\xi)=x$, and then there is a $p \in G$ that forces $\dot{f}$ is a function and $\dot{f}(\check{\xi})=\check{x}$; so $x$ is in the range of $g$. Thus, $g$ maps a subset of $P \times \alpha$ onto a superset of $a$, and therefore $M$ satisfies SVC with $P$.

It is an open problem whether it is consistent with ZF that every limit ordinal has cofinality $\omega$. The following theorem expresses the intuitively obvious idea that any model for this would have to involve a proper class of 
separate violations of choice. (It would also have to satisfy " 0 " exists", by work of Jensen [6].) This fact will be needed in the proof of Theorem 5.1.

THEOREM 4.7. SVC implies that, for any set $A$, there is a limit ordinal $\alpha$ such that $A$ cannot be mapped onto a cofinal subset of $\alpha$.

Proof. Assume SVC in the universe. Pass to a (Boolean-valued) generic extension in which choice holds, by Theorem 4.6. Let $\alpha$ be the next cardinal, in the sense of the extension, after the cardinal of $A$, in the sense of the extension. Then there is no map in the extension from $A$ onto a cofinal subset of $\alpha$. A fortiori, there is no such map in the original universe.

5. Enough injective abelian groups. This section is devoted to the proof of a single theorem which implies that the results of $\$ 3$ could not have been obtained with models of the usual sorts discussed in Theorems 4.2 through 4.5.

\section{THEOREM 5.1. SVC implies EIAb.}

Proof. We begin by establishing some lemmas. The first is a very weak form of EIAb that requires no choice at all. All groups mentioned in this section are assumed to be abelian.

LEMMA 1. Every finitely generated group has an extension $D$ such that, whenever $A \subseteq B$ are finitely generated groups, every homomorphism $A \rightarrow D$ extends to a homomorphism $B \rightarrow D$.

Proof. No choice is needed in the standard proof [20] that every finitely generated group is a direct sum of cyclic groups and can therefore be embedded in a (denumerable) divisible abelian group $D$, a direct sum of copies of the rationals and of the rationals modulo integers. The usual proof (with AC) that divisible groups are injective suffices to prove (without AC) that $D$ has the property required in the lemma.

Alternate PROOF. The statement of the lemma is clearly provable from $\mathrm{AC}$, even if we strengthen it by demanding that $D$ be denumerable. But this strengthened form of the statement is $\Pi_{4}^{1}$ over the natural numbers, so AC can be eliminated from its proof, by Theorem $\mathrm{C}$ of [17].

Lemma 2. Let $G$ be a group, and let $\Gamma$ be a set of triples $(A, B, f)$, where $A \subseteq B$ are groups and $f: A \rightarrow G$ is a homomorphism. There is a group $G^{\prime} \supseteq G$ such that, for each $(A, B, f) \in \Gamma$, there is a homomorphism $f^{\prime}: B \rightarrow G^{\prime}$ that extends $f$. Furthermore, there is a function $\Phi_{G, \Gamma}$ that assigns to each $(A, B, f)$ such an $f^{\prime}$. Finally, $G^{\prime}$ and $\Phi_{G, \Gamma}$ are uniformly definable from $G$ and $\Gamma$.

"Uniformly" means that the same set-theoretic formula defines $G$ ' from $G$ and $\Gamma$, for all $G$ and $\Gamma$; and similarly for $\Phi_{G, \Gamma}$. 
Proof. $G^{\prime}$ is the group generated by $G$ together with formal symbols $(A, B, f, b)$, where $(A, B, f) \in \Gamma$ and $b \in B$, subject to the following relations, in which we abbreviate $(A, B, f, b)$ as $f^{\prime} b$.

(i) $f^{\prime} b_{1}+f^{\prime} b_{2}=f^{\prime}\left(b_{1}+b_{2}\right)$ for any $(A, B, f) \in \Gamma$ and any $b_{1}, b_{2} \in B$,

(ii) $f^{\prime} a=f(a)$ for any $(A, B, f) \in \Gamma$ and any $a \in A$,

(iii) $g_{1}+g_{2}=g$ if, in $G, g_{1}+g_{2}=g$.

In order that $G^{\prime}$ be definable from $G$ and $\Gamma$, we insist that it be constructed in the standard way, so an element of $G^{\prime}$ is an equivalence class of words in the generators. There is obviously a homomorphism $i: G \rightarrow G^{\prime}$, sending each $g \in G$ to (the equivalence class of) the generator $g \in G^{\prime}$. Furthermore, for $(A, B, f) \in \Gamma$, there is a homomorphism $f^{\prime}: B \rightarrow G^{\prime}$ sending each $b \in B$ to (the equivalence class of) the generator $f^{\prime} b=(A, B, f, b)$ of $G^{\prime}$. The restriction of $f^{\prime}$ to $A$ is $i \circ f$. If we show that $i$ is monic, then we can identify $G$ with its image in $G^{\prime}$ under $i$, and the lemma will be proved, because the whole construction is clearly uniformly definable from $G$ and $\Gamma$.

Suppose $i$ were not monic. Then there would be a nonzero $g \in G$ such that the equation $g=0$ is deducible (by linear combination) from finitely many of the equations (i), (ii), (iii). Fix such a deduction of $g=0$. Let $G_{0}$ be the subgroup of $G$ generated by those elements of $G$ that are mentioned in this deduction. Since $G_{0}$ is finitely generated, let $D$ be an extension of it as in Lemma 1. For each $(A, B, f) \in \Gamma$ involved in an equation of type (i) or (ii) used in the deduction, let $A_{0}$ and $B_{0}$ be the subgroups of $A$ and $B$ generated by the finitely many elements that occur in the deduction, and let $f^{*}: B_{0} \rightarrow D$ be an extension of $f \uparrow A_{0}: A_{0} \rightarrow G \hookrightarrow D$; such $f^{*}$ 's exist by our choice of $D$, and there are only finitely many $(A, B, f)$ involved, so we can select the $f^{* \prime}$ 's without invoking AC. Now all the equations used in the deduction become true in $D$ if we interpret $f^{\prime} b$ as $f^{*}(b)$. Therefore, $g=0$ is true in $D$. This is absurd, as $g \neq 0$ in $G_{0} \subseteq D$.

Note that the $G^{\prime}$ of Lemma 2 need not be injective, since $\Gamma$ is only a set.

LEMMA 3. Assume SVC. Let $G$ be a group and $\Gamma$ a set of pairs $(A, B)$ where $A \subseteq B$ are groups. Then there is a group $G^{*}$ such that, if $(A, B) \in \Gamma$, then every homomorphism $f: A \rightarrow G^{*}$ has an extension $f^{*}: B \rightarrow G^{*}$. Furthermore, there is a function $\Psi_{G, \Gamma}$ assigning to each such $(A, B, f)$ an appropriate $f^{*}$. Finally, $G^{*}$ and $\Psi_{G, \Gamma}$ are uniformly definable from $G$ and $\Gamma$.

The key difference between this lemma and the preceding one is that homomorphisms into $G^{*}$, not merely into $G$, are now to be extended. As might be expected, the proof is an iteration of Lemma 2.

Proof. Apply Theorem 4.7 to the union of the sets $A$ for all $(A, B) \in \Gamma$, obtaining a limit ordinal $\alpha$ such that $A$ cannot be mapped cofinally into $\alpha$ for any $(A, B) \in \Gamma$. We define, for each ordinal $\xi \leqslant \alpha$, a group $G(\xi)$ by 
induction on $\xi$. Set $G(0)=G$. If $G(\xi)$ has been defined, let $G(\xi+1)$ be the group $G^{\prime}$ obtained by applying Lemma 2 to $G(\xi)$ and

$$
\Gamma(\xi)=\{(A, B, f) \mid(A, B) \in \Gamma \text { and } f \text { is a homomorphism } A \rightarrow G(\xi)\} .
$$

(The last assertion of Lemma 2 shows that no choice is needed here.) For limit ordinals $\lambda$, set $G(\lambda)=\cup_{\xi<\lambda} G(\xi)$. We claim that $G(\alpha)$ is the desired $G^{*}$.

If $(A, B) \in \Gamma$ and $f: A \rightarrow G(\alpha)$ is a homomorphism, then the function assigning to each $a \in A$ the smallest $\xi$ for which $f(a) \in G(\xi)$ cannot map onto a cofinal subset of $\alpha$, so $f$ maps $A$ into $G(\xi)$ for some $\xi<\alpha$. Then $(A, B, f) \in \Gamma(\xi)$, and we set $\Psi_{G, \Gamma}(A, B, f)=\Phi_{G(\xi), \Gamma(\xi)}(A, B, f)$, an extension of $f$ to a homomorphism $B \rightarrow G(\xi+1) \hookrightarrow G(\alpha)$. That $G^{*}$ and $\Psi_{G, \Gamma}$ are uniformly definable from $G$ and $\Gamma$ is clear from the preceding, provided $\alpha$ is chosen as small as possible.

We are now in a position to prove Theorem 5.1. Assume that SVC holds with $S$, and let $\Gamma$ consist of all pairs $(A, B)$, where $B$ is a quotient group of the free group generated by $S$ and where $A$ is a subgroup of $B$. We use "quotient" in the strict sense of a group of cosets, not merely a homomorphic image, and similarly for "free group"; thus $\Gamma$ is a set. For any given group $G$, we apply Lemma 3 to $G$ and $\Gamma$ obtaining a group $G^{*} \supseteq G$ and an operator $\Psi=\Psi_{G, \Gamma}$ that assigns to every homomorphism $f: A \rightarrow G^{*}$ an extension $\Psi(f)=\Psi(A, B, f): B \rightarrow G^{*}$ when $(A, B) \in \Gamma$. We shall show that $G^{*}$ is injective, thereby completing the proof.

Let $A \subseteq B$ be arbitrary groups, and let $f: A \rightarrow G^{*}$ be an arbitrary homomorphism. We seek to extend $f$ to a homorphism $B \rightarrow G^{*}$. Since SVC holds with $S$, there is a function $e$ from $S \times \alpha$ onto $B$, for some ordinal $\alpha$; fix such $\alpha$ and $e$. For $\xi \leqslant \alpha$, let $B_{\xi}$ be the subgroup of $B$ generated by $A \cup e(S \times \xi)$; in particular, $B_{0}=A$ and $B_{\alpha}=B$. We shall define, for each $\xi \leqslant \alpha$, a homomorphism $f_{\xi}: B_{\xi} \rightarrow G^{*}$ in such a way that $f_{\xi}$ is an extension of $f_{\eta}$ for $\eta<\xi$ and $f_{0}=f$. Then $f_{\alpha}$ will be the required extension of $f$ to $B$. As indicated above, we set $f_{0}=f$, and for limit ordinals $\lambda$ we set $f_{\lambda}=\cup_{\xi<\lambda} f_{\xi}$. All that remains is to define $f_{\xi+1}$, in a uniform way, given $f_{\xi}: B_{\xi} \rightarrow G^{*}$. By definition, $B_{\xi+1}$ is generated by $B_{\xi} \cup X$, where $X=e(S \times\{\xi\})$. The function $s \mapsto e(s, \xi)$ maps $S$ onto $X$ and extends to a homomorphism of the free group generated by $S$ onto the subgroup $\bar{X}$ of $B$ generated by $X$. It therefore induces an isomorphism $i$ from a quotient $Q$ of this free group onto $\bar{X}$. Since $\left(i^{-1}\left(B_{\xi} \cap \bar{X}\right), Q\right)$ belongs to the $\Gamma$ used in constructing $G^{*}$, we can apply $\Psi$ to the homomorphism $f_{\xi} \circ i: i^{-1}\left(B_{\xi} \cap \bar{X}\right) \rightarrow G^{*}$, obtaining an extension $Q \rightarrow G^{*}$. Composing with $i^{-1}$, we have a homomorphism $g=$ $i^{-1} \circ \Psi\left(f_{\xi} \circ i\right)$ from $\bar{X}$ to $G^{*}$ that agrees with $f_{\xi}$ on $B_{\xi} \cap \bar{X}$. Since $f_{\xi}$ and $g$ agree on the intersection of their domains, they define a homomorphism into $G^{*}$ from the subgroup of $B$ generated by their domains. But this subgroup is $B_{\xi+1}$, so we have the required $f_{\xi+1}$. We have given the definition of $f_{\xi+1}$ in 
more detail than would otherwise be necessary, in order to emphasize that, once $e$ is fixed, no arbitrary choices were needed, so the inductive definition of the $f_{\xi}$ 's can be carried out in the absence of AC.

Combining Theorem 5.1 with Theorems 4.2 through 4.5 , we see that EIAb holds in all permutation models, all symmetric models, and all models of the forms $L(A)$, with $A$ a transitive set, and $\operatorname{HOD}(A)$, with $A$ an arbitrary set. Since models of these sorts were used to prove a great variety of independence results involving weak axioms of choice (see [10]), we conclude that all these independences continue to hold in the presence of EIAb. In particular, none of the weak axioms of choice mentioned in the appendix of [10] can be derived from EIAb.

6. Enough projective sets. The main result is that, as a weak axiom of choice, EPSets is at the opposite end of the spectrum from EIAb. In "ordinary" models of the negation of AC, EIAb always holds, by Theorem 5.1, and EPSets never holds.

THEOREM 6.1. EPSets and SVC together imply AC.

PROoF. If SVC holds with $S_{1}$ and if $S_{2}$ can be mapped onto $S_{1}$, then clearly SVC holds with $S_{2}$ also. Thus, the two assumptions of the theorem imply that SVC holds with a projective set $S$. Fix such an $S$. Let $Z$ be any set; we shall prove that $Z$ admits a well-ordering.

Let $g$ map $S \times \alpha$ onto the set $Z^{S}$ of all functions from $S$ into $Z$, for some ordinal $\alpha$. For each $s \in S$, let

$$
Z_{s}=\{g(s, \xi)(s) \mid \xi<\alpha\}
$$

Each $Z_{s}$, being defined as the image of $\alpha$ under some function, obviously admits a well-ordering, so it suffices to prove that $Z_{s}=Z$ for some $s \in S$. Suppose not. Then, as $S$ is projective, there is a choice function $f: S \rightarrow Z$ with $f(s) \in Z-Z_{s}$ for each $s \in S$. By definition of $g$, there exist $s \in S$ and $\xi<\alpha$ such that $f=g(s, \xi)$. But then, for this $s, f(s)=g(s, \xi)(s) \in Z_{s}$, a contradiction.

As in the discussion at the end of $\$ 5$, we infer from Theorem 6.1 that all the usual independence results involving weak axioms of choice remain true in the presence of the negation of EPSets. In particular, none of the axioms in the appendix of [10], except AC itself, implies EPSets.

The following result was obtained independently by Aczel [1]; we include it here for the sake of completeness, because it is essentially all that is known about the strength of EPSets in the absence of SVC.

THEOREM 6.2. EPSets implies the axiom of dependent choice. 
Proof. Let $R$ be a binary relation on a set $A$ such that $\forall x \in A \exists y \in A$ $R(x, y)$, and let $a \in A$. By EPSets let $p: P \rightarrow A$ be a map of a projective set $P$ onto $A$. Since $P$ is projective, there is a function $g: P \rightarrow P$ such that $\forall x \in P$ $R(p(x), p g(x))$. If $b$ is any point in $p^{-1}\{a\}$, then the function $f(n)=p g^{n}(b)$ satisfies $\forall n \in \omega R(f(n), f(n+1))$ and $f(0)=a$, as required by the axiom of dependent choice.

As we mentioned above, very little is known about the strength of EPSets. In particular, we do not know whether it implies AC (without assuming SVC); we conjecture that it does not. In connection with this conjecture, it is perhaps worthwhile to point out that the most naive approach to refuting it, namely to claim that the surjective image of a projective set is itself projective, fails.

EXAMPLE 6.3. A nonprojective image of a projective set.

We use the following "mixture" of the first and second Fraenkel models. Let $A$ be a denumerable set of atoms, $a_{n}$ and $b_{n}(n \in \omega)$. Let $P_{n}=\left\{a_{n}, b_{n}\right\}$. Let $\mathcal{G}$ be the group of permutations of $A$ that preserve the partition $\left\{P_{n} \mid n \in\right.$ $\omega$ ), i.e., if $a_{n}$ is sent to $a_{m}$ (resp. $b_{m}$ ) then $b_{n}$ is sent to $b_{m}$ (resp. $a_{m}$ ). Let $\mathscr{F}$ be the filter generated by the groups $H_{E}$ of permutations that fix $E$ pointwise, where $E$ ranges over finite subsets of $A$. We claim that, in the resulting permutation model $M$, the set $A$ of atoms is projective.

Let $p$ map a set $X$ onto $A$ in $M$. Let $E \subseteq A$ be a finite support for $p$ (i.e. if $g \in H_{E}$ then $g(p)=p$ ) and hence also for $X$. We may assume that $E$ is a union of some of the pairs $P_{n}$. Arbitrarily fix some $a_{m} \in A-E$ and some $x \in p^{-1}\left\{a_{m}\right\}$, and let $F$ be a finite union of pairs such that $E \cup P_{m} \cup F$ supports $x$ and $E, P_{m}, F$ are pairwise disjoint. Then

$$
f=\left\{\left(g a_{m}, g x\right) \mid g \in H_{E \cup F}\right\}
$$

is easily seen to be in $M$ and to be a function, by our choice of $F$. By our choice of $E, p(x)=a_{m}$ implies $p(g x)=g a_{m}$, so $f$ is a partial section of the projection $p$. Its domain is $A-E-F$. Since $E \cup F$ is finite, $f$ can be extended to a section of $p$ defined on all of $A$. Therefore, $A$ is projective.

The map sending each atom to the pair $P_{n}$ that contains it is in $M$ and maps $A$ onto the set $\left\{P_{n} \mid n \in \omega\right\}$. This (Dedekind finite) set is not projective in $M$, because no function in $M$ selects one atom from each $P_{n}$. Thus, we have a projective set $A$ mapping onto a nonprojective set.

We conclude this section by commenting briefly on an interesting weakening of EPSets. By a resolution of a set $x$, we mean a function $f$ from some set $y$ onto $x$, such that, for any function $g$ from any set $z$ onto $x$, there is a map $h: y \rightarrow z$ such that $f=g h$. Thus, for example, $x$ is projective if and only if its identity map is a resolution. In general, a resolution of $x$ intuitively represents the "worst possible" failure of $x$-indexed choice.

We consider the principle "Every set has a resolution"; it follows 
immediately from EPSets because every map of a projective set onto $x$ is a resolution of $x$. The only nontrivial fact known to me about this principle is that, in permutation models, it implies well-ordered choice, i.e., all ordinals are projective, but not conversely. The proof has more length than interest, so we omit it, but we mention that the "not conversely" part is proved by using the permutation model obtained from $\aleph_{1}$ atoms, the group of all permutations, and the filter determined by countable supports. This model satisfies well-ordered choice (Theorem 8.9 of [10]), but the set of countable sequences of atoms has no resolution in the model.

7. Enough projective abelian groups. We shall apply the methods used in the proofs of Theorems 6.1 and 6.2 to EPAb and the slightly stronger principle

EFPAb. Every abelian group is a homomorphic image of a free projective abelian group.

Note that the usual proof that projective abelian groups are free uses the axiom of choice, and Example 7.1 below shows that this use is essential. Thus, EFPAb and EPAb are not trivially equivalent. We conjecture that they are not equivalent at all.

EXAMPLE 7.1. A projective abelian group that is not free.

We use a variation of Example 6.3. Let $G_{0}$ be a finite group; we shall impose some conditions on $G_{0}$ later, but for the moment it can be arbitrary. Let $V$ be a model of ZFCA whose atoms form a set in one-to-one correspondence with $G_{0} \times \omega$; fix such a correspondence, and write $\langle g, n\rangle$ for the atom that corresponds to the pair $(g, n) . G_{0}$ is assumed to be a pure set, i.e., to involve no atoms. Any permutation $\pi$ of $\omega$ acts on $A$ by sending $\langle g, n\rangle$ to $\langle g, \pi(n)\rangle$. Any $\omega$-sequence $\left(g_{0}, g_{1}, \ldots\right)$ of elements of $G_{0}$ acts on $A$ by sending $\langle g, n\rangle$ to $\left\langle g_{n} g, n\right\rangle$. Let $\mathcal{G}$ be the group generated by all permutations of these two sorts (a wreath product). Let $\mathscr{F}$ be the filter generated by the subgroups $H_{E}$ fixing $E$ pointwise, where $E$ ranges over finite subsets of $A$. Note that it would make no difference if $E$ ranged only over cylinders, i.e., subsets of $A$ that correspond to subsets $G_{0} \times F \subseteq G_{0} \times \omega$, where $F$ is a finite subset of $\omega$. Let $M$ be the permutation submodel of $V$ defined by $\mathcal{G}$ and $\mathscr{F}$.

The set $A$ of atoms is projective in $M$; this is proved exactly as in Example 6.3. By the proof of Lemma 1.1(a) it follows that the free abelian group $\mathbf{Z} A$ generated by $A$ is projective.

The partition $\Pi$ of $A$ into subsets (minimal cylinders)

$$
k^{*}=\left\{\langle g, k\rangle \mid g \in G_{0}\right\},
$$

corresponding to $G_{0} \times\{k\}$, is in $M$, but its enumeration in an $\omega$-sequence, $k \mapsto k^{*}$, is not; in fact, $\Pi$ is Dedekind finite in $M$. The obvious bijections $\theta_{k^{*}}$ : $k^{*} \rightarrow G_{0}:\langle g, k\rangle \mapsto g$ are in $M$, being finite, but the function assigning $\theta_{k^{*}}$ to $k^{*}$ is not in $M$. Let $\Theta_{k^{*}}$ be the set of bijections from $k^{*}$ to $G_{0}$ obtained by composing $\theta_{k^{*}}$ with left translations of $G_{0}$; thus a typical element of $\Theta_{k^{*}}$ is 


$$
h \circ \theta_{k^{*}}: k^{*} \rightarrow G_{0}:\langle g, k\rangle \mapsto h g
$$

with $h \in G_{0}$. It is easy to check that the function $k^{*} \mapsto \Theta_{k^{*}}$ is in $M$.

Since $A$ is the disjoint union of the sets $k^{*}, \mathbf{Z} A$ is the direct sum of the groups $\mathbf{Z} k^{*}$ freely generated by $k^{*}$. Let $k^{*}$-pr: $\mathbf{Z} A \rightarrow \mathbf{Z} k^{*}$ be the projection obtained from this direct sum decomposition. Composing with the isomorphism $\bar{\theta}_{k^{*}}: \mathbf{Z} k^{*} \rightarrow \mathbf{Z} G_{0}$ induced by $\boldsymbol{\theta}_{k^{*}}$ or its composites $\overline{h \theta_{k^{*}}}$ with left translations, we get a set $\Gamma_{k^{*}}$ of projections $\mathbf{Z} A \rightarrow \mathbf{Z} G_{0}$; the function sending $k^{*}$ to $\Gamma_{k^{*}}$ is in $M$.

Recall that the multiplication operation of $G_{0}$ makes $Z G_{0}$ a ring, the integral group ring of $G_{0}$. We shall establish a connection between its left modules and subgroups of powers of $\mathbf{Z} A$ in $M$. Specifically, let $n$ be a positive integer, and let $P_{0}$ be a $\mathbf{Z} G_{0}$-submodule of the free module $\left(\mathrm{ZG}_{0}\right)^{n}$. Set

$$
\begin{aligned}
& P=\left\{x \in(\mathbf{Z} A)^{n} \mid \text { for all } k^{*} \in \Pi\right. \text { and some (or all) } \\
& \left.\qquad \alpha \in \Gamma_{k^{*}}, \alpha^{(n)}(x) \in P_{0}\right\} .
\end{aligned}
$$

Here $\alpha^{(n)}:(\mathbf{Z} A)^{n} \rightarrow\left(\mathbf{Z} G_{0}\right)^{n}$ is defined by $\alpha: \mathbf{Z} A \rightarrow \mathbf{Z} G_{0}$ componentwise. Note that it makes no difference whether we refer to some $\alpha \in \Gamma_{k^{*}}$ or to all $\alpha \in \Gamma_{k^{*}}$ in defining $P$; this is because the various $\alpha$ 's differ only by left translations and $P_{0}$ is invariant under these. It is clear that $P$ is a subgroup of $(\mathbf{Z} A)^{n}$, and it is in $M$ because only elements of $M$ were used in its definition. (This is the reason for using $\Gamma_{k^{*}}$ rather than $\bar{\theta}_{k^{*}} \circ k^{*}$-pr; the latter, as a function of $k^{*}$, is not available in $M$.) Note that, if $Q_{0}$ is a submodule of $\left(\mathbf{Z} G_{0}\right)^{n}$ complementary to $P_{0}$, i.e., if $P_{0} \oplus Q_{0}=\left(Z G_{0}\right)^{n}$, then the corresponding subgroups of $(\mathbf{Z} A)^{n}$ are also complementary, $P \oplus Q=(\mathbf{Z A})^{n}$. (This can be checked in $V$ rather than $M$, and there it is clear since $\mathbf{Z} A, P, Q$ are just the $\omega$-fold direct sums of $\mathbf{Z} G_{0}, P_{0}, Q_{0}$ respectively.)

We now require $G_{0}$ to be such that some finitely generated projective $\mathrm{Z} G_{0}$-module $P_{0}$ is not free. $\operatorname{Rim}[18]$ showed that such groups exist and in fact that the cyclic group of order 23 will do. Being finitely generated and projective, $P_{0}$ is a direct summand of a finitely generated free module, say $\left(\mathrm{Z} G_{0}\right)^{n}=P_{0} \oplus Q_{0}$. Then the associated subgroup $P$ of $(\mathbf{Z} A)^{n}$ is, in $M$, a direct summand of $(\mathbf{Z} A)^{n}$, and is therefore projective in $M$, because $\mathbf{Z} A$ is.

We show that $P$ is not free in $M$. Suppose $B \in M$ were a free basis for $P$. Being hereditarily symmetric, $B$ is supported by the union of finitely many $k^{*}$ 's; let $S$ be the set of these $k^{*}$ 's, and fix some $m^{*} \in \Pi-S$. Since $B$ generates $P$,

$$
B^{\prime}=\left\{\bar{\theta}_{m^{*}} \circ m^{*}-\operatorname{pr}(b) \mid b \in B, m^{*}-\operatorname{pr}(b) \neq 0\right\}
$$

generates $\bar{\theta}_{m^{*}} \circ m^{*}-\operatorname{pr}(P)=P_{0}$ as an abelian group.

We claim that $B^{\prime}$ is invariant under the action of $G_{0}$ on $P_{0}$. To see this, take any $h \in G_{0}$, and consider the permutation $\sigma$ of $A$ that fixes $A-m^{*}$ point- 
wise and sends each $\langle g, n\rangle$ to $\langle h g, m\rangle$. This permutation is in $\mathcal{G}$ and fixes the support of $B$ pointwise, so it fixes $B$. Thus, for any element $\bar{\theta}_{m^{*}} \circ m^{*}-\operatorname{pr}(b)$ of $B^{\prime}$, with $b \in B$, we have $\sigma(b) \in B$, so $B^{\prime}$ contains the element

$$
\begin{aligned}
\bar{\theta}_{m^{*}} \circ m^{*}-\operatorname{pr}(\sigma(b)) & =\bar{\theta}_{m^{*}} \circ \sigma \circ m^{*}-\operatorname{pr}(b) \\
& =h \circ \bar{\theta}_{m^{*}} \circ m^{*}-\operatorname{pr}(b),
\end{aligned}
$$

as claimed.

We claim that $B^{\prime}$ is not linearly independent over $\mathbf{Z}$. For suppose it were. Then $P_{0}$ would be the direct sum of the free abelian groups $\mathbf{Z X}$ generated by the $G_{0}$-orbits $X$ in $B^{\prime}$. Each of these summands is also a $Z G_{0}$-module, and in fact a projective one, because $P_{0}$ is projective. If $G_{0}$ acted freely on every orbit $X$, then each $\mathbf{Z} X$ would be free over $\mathbf{Z} G_{0}$, which is absurd because their sum $P_{0}$ is not free. So consider an orbit $X$ where the action of $G_{0}$ is not free; let $H$ be the isotropy group of some point $x \in X$. So $H$ is not the trivial group. Consider the map $G_{0} \rightarrow X: g \mapsto g x$; since it is surjective, it induces an epimorphism $e: \mathbf{Z} G_{0} \rightarrow \mathbf{Z} X$ which admits a splitting $\mathbf{Z} G_{0}$-homomorphism $s$ : $\mathbf{Z} X \rightarrow \mathbf{Z} G_{0}$ because $\mathbf{Z} X$ is projective. Since $H$ fixes $x$, it fixes $s(x)$ as well, so, in particular, all elements of $H$ occur with the same integer coefficient in $s(x)$. On the other hand, the sum of these coefficients is 1 , because es $(x)=x$. This is possible only if $H$ has only one element, a contradiction, which proves that $B^{\prime}$ is linearly dependent over $\mathbf{Z}$.

Take a nontrivial linear dependence between elements of $B^{\prime}$, say

$$
0=\sum_{i=1}^{r} c_{i} \bar{\theta}_{m^{*}} \circ m^{*}-\operatorname{pr}\left(b_{i}\right)
$$

with $b_{i} \in B$ and $c_{i} \in \mathbf{Z}$ and all $m^{*}-\operatorname{pr}\left(b_{i}\right) \neq 0$. Since $\bar{\theta}_{m^{*}}$ is an isomorphism, the element

$$
a=\sum_{i=1}^{r} c_{i} b_{i}
$$

of $B$ has $m^{*}-\operatorname{pr}(a)=0$. Choose $l^{*} \in \Pi-S-\left\{m^{*}\right\}$ such that $l^{*}-\operatorname{pr}\left(b_{i}\right)=0$ for each $i$; since $b_{i} \in \mathbf{Z} A$ has only finitely many nonzero components, there are many such $l^{*}$. Let $\sigma \in \mathcal{G}$ be the permutation that interchanges $\langle g, m\rangle$ with $\langle g, l\rangle$ for all $g$ and fixes $A-m^{*}-l^{*}$ pointwise. Then, since $m^{*}-\operatorname{pr}(a)$ $=l^{*}-\operatorname{pr}(a)=0, \sigma$ fixes $a$. Since $\sigma$ fixes the support of $B$ pointwise, it fixes $B$. In particular, each $\sigma\left(b_{i}\right)$ is in $B$. The definition (1) of $a$ implies

$$
a=\sigma(a)=\sum_{i=1}^{r} c_{i} \sigma\left(b_{i}\right) .
$$

Since $m^{*}-\operatorname{pr}\left(b_{i}\right) \neq 0$, we find that $l^{*}-\operatorname{pr}\left(\sigma\left(b_{i}\right)\right) \neq 0$. Thus, all the $\sigma\left(b_{i}\right)$ are distinct from all the $b_{i}$. But then, by subtracting (1) from (2), we obtain a nontrivial linear dependence between elements of $B$, contradicting the 
assumption that $B$ is a free basis for $P$. Therefore $P$, although projective, is not free in $M$.

Before proceeding to the main results of this section, we introduce two weak axioms of choice that occur in these results. The first is the well-known axiom of multiple choice.

MC. For any family of nonempty sets, there is a function that assigns to each of these sets a nonempty finite subset.

Recall [10, Chapter 9] that MC implies AC in ZF but not in ZFA. We shall need the following lemma, whose proof we omit since it is a trivial variation of the usual proof that $\mathrm{AC}$ is equivalent to the well-ordering principle.

LEMMA 7.2. MC holds if and only if every set is the union of a well-ordered family of finite sets.

The second axiom we shall need is related to the axiom of dependent choice as $\mathrm{MC}$ is related to $\mathrm{AC}$. To formulate this axiom of dependent multiple choice, we define a tree to be a partially ordered set in which (i) there is a (unique) smallest element, called the root, (ii) the predecessors of each point form a finite linearly ordered set, and (iii) every point has at least one immediate successor. The $n$th level of a tree is the set of points that have exactly $n$ strict predecessors. A subtree of a tree is a subset that is a tree with the induced ordering and is closed under predecessor. The axiom of dependent choice is equivalent to the assertion that every tree has a path, i.e., a subtree with exactly one point per level. (The less trivial of the two implications is proved by using a tree of finite approximations to dependent choice sequences.) We are now ready to formulate the axiom of dependent multiple choice.

DMC. Every tree has a subtree whose levels are finite.

Clearly, dependent choice and multiple choice each imply DMC. Also, DMC together with the axiom of choice from countably many finite sets implies dependent choice, for we can, using choice from countably many finite sets, simultaneously well-order all the levels of a tree with finite levels, and then the usual proof of König's infinity lemma provides a path through any such tree.

\section{THEOREM 7.3. EPAb implies DMC.}

Proof. Let $T$ be a tree with root $r$, let $\mathbf{Z T}$ be the free abelian group generated by $T$, and let $p: P \rightarrow \mathbf{Z} T$ be an epimorphism with $P$ projective. We define a homomorphism $f: \mathbf{Z T} \rightarrow \mathbf{Z T}$ by its action on generators as follows. The root $r$ is mapped to zero, and all other generators are mapped to their immediate predecessors in $T$. Since every point in $T$ has an immediate successor, $F$ is an epimorphism. Since $P$ is projective, the map $p: P \rightarrow \mathrm{ZT}$ can be lifted along the epimorphism $f p: P \rightarrow \mathbf{Z} T$ to a homomorphism $h: P \rightarrow P$ 
with $f p h=p$. Fix an $x \in P$ such that $p(x)=r$. For each $n$, let $X_{n}$ be the set of points at the $n$th level of $T$ that occur with nonzero coefficient in the element $p h^{n}(x)$ of $\mathbf{Z T}$. Obviously, $X_{n}$ is finite. By choice of $x, X_{0}=\{r\}$. By choice of $h, f p h^{n+1}(x)=p h^{n}(x)$, so every element of $X_{n}$ has an immediate successor in $X_{n+1}$. Thus, the set $T^{\prime}$, consisting of those points of $T$ each of whose predecessors (including itself) is in some $X_{n}$, is a subtree of $T$ with finite levels.

We digress for a moment into topology. The usual proof $[12, \mathrm{p} .115]$ of Urysohn's lemma uses the axiom of dependent choice to successively select open sets separating previously chosen sets. David Pincus has pointed out that the axiom of multiple choice also implies Urysohn's lemma, since one can use the intersection of the finitely many separating open sets provided by MC. Essentially the same argument shows that DMC implies Urysohn's lemma. One applies DMC to a tree whose points at level $n$ are $\left(2^{n}-1\right)$-tuples of open sets with the separation properties required at the $n$th stage of the usual proof of Urysohn's lemma. The immediate predecessor of such a $\left(2^{n}-1\right)$-tuple consists of every second element of it. From the finitely many points at level $n$ in the subtree given by DMC, one forms a single $\left(2^{n}-1\right)$ tuple by intersecting corresponding components. The tuples so obtained have all the properties needed to carry out the rest of the usual proof.

In view of this discussion and Theorem 7.3, we have the following curious connection between algebra and topology.

COROLlaRY 7.4. EPAb implies Urysohn's lemma.

The next theorem is the abelian group analog of Theorem 6.1.

THEOREM 7.5. In ZFA + SVC, multiple choice follows from EFPAb.

COROllaRY 7.6. In ZF + SVC, the axiom of choice follows from EFPAb.

Proof of Theorem 7.5. Let $S$ and $P$ be sets such that SVC holds with $S$, the free abelian group $\mathbf{Z P}$ generated by $P$ is projective, and there is an epimorphism $e: \mathbf{Z P} \rightarrow \mathbf{Z S}$. Let $Z$ be an arbitrary set. By our choice of $S$, there exist an ordinal $\alpha$ and a function $f$ from $S \times \alpha$ onto the set $\mathscr{P}_{<\omega}(Z)^{P}$ of functions from $P$ to finite subsets of $Z$. For each $p \in P$, let

$$
Z_{p}=\bigcup\{f(s, \xi)(p) \mid \xi<\alpha, s \text { occurs in } e(p)\} .
$$

As only finitely many $s \in S$ occur in the expansion of an element $e(p) \in \mathbf{Z} S$, $Z_{p}$ is the union of a well-ordered family of finite subsets of $Z$. Since $Z$ is arbitrary, Lemma 7.2 shows that we will be done if we can show $Z=Z_{p}$ for some $p \in P$. 
Suppose $Z \neq Z_{p}$ for all $p \in P$, and let $Q$ be the disjoint union

$$
Q=\amalg_{p \in P}\left(Z-Z_{p}\right)=\left\{(p, z) \mid p \in P, z \in Z-Z_{p}\right\} .
$$

The projection $(p, z) \mapsto p$ of $Q$ onto $P$ induces an epimorphism $g: \mathbf{Z} Q \rightarrow \mathbf{Z P}$ which has a section $h: \mathbf{Z P} \rightarrow \mathbf{Z} Q$ because $\mathbf{Z P}$ is projective. The function that assigns to each $p \in P$ the finite subset

$$
X_{p}=\left\{z \in Z-Z_{p} \mid(p, z) \text { occurs in } h(p)\right\}
$$

is $f(s, \xi)$ for some $\xi<\alpha$ and some $s \in S$. Since $e$ is surjective, there exists $p \in P$ such that $s$ occurs in $e(p)$. For this $p$, we have

$$
X_{p}=f(s, \xi)(p) \subseteq Z_{p}
$$

by definition of $Z_{p}$. But $X_{p} \subseteq Z-Z_{p}$ by definition, so $X_{p}$ is empty. But this contradicts the fact that $g h(p)=p$.

Instead of assuming that there are no atoms, as in Corollary 7.6, we can achieve the same result by assuming the axiom of choice for countable families.

THEOREM 7.7. In ZFA + SVC, the axiom of countable choice and EFPAb together imply the axiom of choice.

Proof. Assume all the hypotheses, and let $P$ be a set such that $\mathbf{Z P}$ is a projective abelian group. Our first goal is to show that such a set $P$ is necessarily projective.

Consider any function $f$ from an arbitrary set $X$ onto $P$. Since $\mathbf{Z P}$ is projective, the induced epimorphism $\bar{f}: \mathbf{Z} X \rightarrow \mathbf{Z} P$ admits a section $s$. For each $p \in P$, let $X_{p}^{\prime}=\left\{x \in f^{-1}\{p\} \mid x\right.$ has positive coefficient in $\left.s(p)\right\}$. Thus, $X_{p}^{\prime}$ is a finite nonempty subset of $f^{-1}\{p\}$, a proper subset unless $f^{-1}\{p\}$ is a singleton. Thus, we have shown that every $P$-indexed family of sets of cardinality at least two admits a $P$-indexed family of finite nonempty proper subsets.

We show, by induction on $n$, that any $P$-indexed family of nonempty sets $X_{p}$ of cardinality at most $n$ admits a choice function. This is trivial for $n=1$. If $n \geqslant 2$, let $P^{\prime}=\left\{p \in P|| X_{p} \mid=n\right\} . \mathbf{Z} P^{\prime}$ is projective, being a direct summand of $\mathbf{Z} P$, so the result of the preceding paragraph may be applied to produce nonempty subsets $X_{p}^{\prime} \leqslant X_{p}$, with $\left|X_{p}^{\prime}\right| \leqslant n-1$, for $p \in P^{\prime}$. Apply the induction hypothesis to the family consisting of $X_{p}^{\prime}$ for $p \in P^{\prime}$ and $X_{p}$ for $p \in P-P^{\prime}$; the result is the desired choice function.

Consider now an arbitrary $P$-indexed family of finite sets $X_{p}$. For each $n$, let $P_{n}=\left\{p \in P|| X_{p} \mid=n\right\}$. As $\mathbf{Z} P_{n}$ is projective, being a direct summand of $\mathbf{Z} P$, the preceding discussion yields a choice function $f_{n}$ on $P_{n}$. By the axiom of countable choice, we can select one $f_{n}$ for each $n$; the union of these is a choice function on $P$. 
Finally, for a $P$-indexed family of sets that may be infinite, we use the second paragraph of this proof or MC, which holds by Theorem 7.5, to extract finite subsets, and then we apply the result of the preceding paragraph to obtain a choice function. Therefore $P$ is projective, as claimed.

Observe that the product of two projective sets, $P_{1}$ and $P_{2}$, is also projective. For if $\left\{X_{i j} \mid i \in P_{1}, j \in P_{2}\right\}$ is a family of nonempty sets, then the projectivity of $P_{1}$ provides, for each $j \in P_{2}$, a choice function $f_{j}$ with $f_{j}(i) \in$ $X_{i j}$ for all $i \in P_{1}$. The projectivity of $P_{2}$ then lets us choose one such $f_{j}$ for each $j \in P_{2}$, and we define the choice function $f$ on $P_{1} \times P_{2}$ by $f(i, j)=$ $f_{j}(i)$.

The assumption of countable choice asserts that $\omega$ is projective. Therefore, if $\mathbf{Z} P$ is a projective abelian group, then $P \times \omega$ is a projective set, and as a trivial consequence all subsets of $P \times \omega$ are projective.

Let $S$ be any set; use the hypothesis EFPAb to get a set $P$ such that $\mathbf{Z P}$ is projective and admits an epimorphism $e: \mathbf{Z P} \rightarrow \mathbf{Z S}$. As $P$ is projective, by the above, we can choose, for each $p \in P$, a linear ordering $<_{p}$ of the finite set $S_{p}=\{s \in S \mid s$ occurs in $e(p)\}$. By sending the pair $(p, n)$, with $p \in P$ and $n<\left|S_{p}\right|$, to the $n$th element of $S_{p}$ in the ordering $<_{p}$, we define a function from a subset of $P \times \omega$ onto $\cup_{p \in P} S_{p}$, i.e., onto $S$ because $e$ is an epimorphism. Since all subsets of $P \times \omega$ are projective and $S$ is arbitrary, we have obtained EPSet. Since SVC is assumed, Theorem 6.1 gives AC.

We close with one more variation on the theme of deducing AC from the existence of projective abelian groups.

THEOREM 7.8. Assume that SVC holds with $S$ and that $e$ is an epimorphism from a free projective abelian group $\mathrm{ZP}$ onto $\mathrm{ZS}$ that maps $P$ into, and therefore onto $S$. Then the axiom of choice holds.

Proof. Notice that the hypotheses are sufficient to carry out the proof of MC as in Theorem 7.5. By Theorem 9.1 of [10], it follows that every linearly ordered set can be well-ordered.

Let $X$ be an arbitrary set; we intend to well-order it. Since SVC holds with $S$, let $\alpha$ be an ordinal and $f$ a function from $S \times \alpha$ onto $(\mathbf{Z} X)^{P}$. For $p \in P$ and $x \in X$, define an $\alpha$-sequence of integers $\nu(p, x): \alpha \rightarrow Z$ by

$$
\nu(p, x)(\xi)=\text { coefficient of } x \text { in } f(e(p), \xi)(p) .
$$

Since the set ${ }^{\alpha} \mathbf{Z}$ of $\alpha$-sequences of integers can be linearly ordered lexicographically, MC provides a well-ordering of it. Fix such a well-ordering. If, for some $p \in P$, the map $x \mapsto \nu(p, x)$ of $X$ into ${ }^{\alpha} Z$ is one-to-one, then $X$ inherits a well-ordering from ${ }^{\alpha} \mathbf{Z}$, and we are done. Otherwise, we obtain a contradiction as follows. 
For each $p \in P$, let $\nu(p)$ be the first element of ${ }^{\alpha} Z$ (in the fixed wellordering) that is $\nu(p, x)$ for at least two distinct $x \in X$, and let $X_{p}=\{x \in$ $X \mid \nu(p, x)=\nu(p)\}$. So $\left|X_{p}\right| \geqslant 2$ for all $p \in P$. Let

$$
Q=\coprod_{p \in P} X_{p}
$$

and let $g$ be the canonical projection $Q \rightarrow P:(p, x) \mapsto p$. It induces an epimorphism $\bar{g}: \mathbf{Z} Q \rightarrow \mathbf{Z} P$, which has a section $h: \mathbf{Z} P \rightarrow \mathbf{Z} Q$ because $\mathbf{Z} P$ is projective. Define $h^{\prime}: P \rightarrow \mathbf{Z} X$ by

$$
h^{\prime}(p)=\text { the component in } \mathbf{Z} X_{p} \text { of } h(p) ;
$$

here we use the canonical identification of $\mathrm{Z} Q$ with the direct sum $\bigoplus_{p \in P} \mathbf{Z} X_{p}$. Note that, because $\bar{g} h(p)=p$, the sum of the coefficients of all the terms in $h^{\prime}(p)$ is 1 .

Since $h^{\prime}$ maps $P$ into $\mathbf{Z} X$, it is $f(s, \xi)$ for some $s \in S$ and some $\xi<\alpha$. And $s=e(p)$ for some $p \in P$; fix such $s, \xi$, and $p$. For all $x \in X_{p}$, we have

$$
\text { coefficient of } \begin{aligned}
x \text { in } h^{\prime}(p) & =\text { coefficient of } x \text { in } f(e(p), \xi)(p) \\
& =\nu(p, x)(\xi)=\nu(p)(\xi)
\end{aligned}
$$

which is independent of $x \in X_{p}$. So the sum of all the coefficients in $h^{\prime}(p)$ is divisible by $\left|X_{p}\right| \geqslant 2$. This contradicts the fact that this sum is 1 .

\section{REFERENCES}

1. P. Aczel, Interpretations of constructive set theory, (Logic Colloquium, 1977, Wrocław), North-Holland, Amsterdam.

2. M. K. Armbrust, An algebraic equivalent of a multiple choice axiom, Fund. Math. 74 (1972), $145-146$.

3. A. Blass, Two algebraic equivalents of the axiom of choice, Notices Amer. Math. Soc. 22 (1975), A-524.

4. H. Cartan and S. Eilenberg, Homological algebra, Princeton Univ. Press, Princeton, N. J., 1956.

5. P. J. Cohen, Set theory and the continuum hypothesis, Benjamin, New York, 1966.

6. K. Devlin and R. B. Jensen, Marginalia to a theorem of Silver, Proc. Logic Conference, Kiel, 1974 (ed. by G. H. Müller, A. Oberschelp, and K. Potthoff), Lecture Notes in Math., vol. 499, Springer-Verlag, Berlin and New York, 1975, pp. 115-142.

7. W. B. Easton, Powers of regular cardinals, Ann. Math. Logic 1 (1970), 139-178.

8. S. Grigorieff, Intermediate submodels and generic extensions in set theory, Ann. of Math. (2) 101 (1975), 447-490.

9. W. Hodges, private communication.

10. T. Jech, The axiom of choice, North-Holland, Amsterdam, 1973.

11. R. B. Jensen, Modelle der Mengenlehre, Lecture Notes in Math., vol. 37, Springer-Verlag, Berlin and New York, 1967.

12. J. L. Kelley, General topology, Van Nostrand, Princeton, N. J., 1955.

13. A. Levy, Definability in axiomatic set theory. I, Proc. Int. Congress on Logic, Methodology, and Philosophy of Science, 1964 (ed. by Y. Bar-Hillel), North-Holland, Amsterdam, 1965.

14. S. MacLane, Categories for the working mathematician, Springer-Verlag, New York, 1971. 
15. D. Morris, $A$ model of $\mathrm{ZF}$ which cannot be extended to a model of $\mathrm{ZFC}$ without adding ordinals, Notices Amer. Math. Soc. 17 (1970), 577.

16. D. G. Northcott, An introduction to homological algebra, Cambridge Univ. Press, Cambridge, 1960.

17. R. Platek, Eliminating the continuum hypothesis, J. Symbolic Logic 34 (1969), 219-225.

18. D. S. Rim, Modules over finite groups, Ann. of Math. 69 (1959), 700-712.

19. J. R. Shoenfield, Unramified forcing, Axiomatic Set Theory, (ed. by D. Scott), Proc. Sympos. Pure Math., vol. 13, Part I, Amer. Math. Soc., Providence, R. I., 1971, pp. 357-381.

20. B. L. van der Waerden, Algebra. II, 5th ed., Springer-Verlag, Berlin, 1967.

Department of Mathematics, University of Michigan, ANn Arbor, Michigan 48109 